\title{
Effect of alcohol consumption on food energy intake: a systematic review and meta-analysis
}

\author{
Alastair Kwok ${ }^{1 *}$, Aimee L. Dordevic ${ }^{1}$, Gemma Paton ${ }^{1}$, Matthew J. Page ${ }^{2}$ and Helen Truby ${ }^{1}$ \\ ${ }^{1}$ Department of Nutrition, Dietetics and Food, Monash University, School of Clinical Sciences, Notting Hill, VIC 3168, Australia \\ ${ }^{2}$ School of Public Health and Preventive Medicine, Monash University, Melbourne, VIC 3004, Australia \\ (Submitted 9 March 2018 - Final revision received 23 October 2018 - Accepted 14 November 2018 - First published online 29 January 2019)
}

\section{Abstract}

The relationship between alcohol consumption and body weight is complex and inconclusive being potentially mediated by alcohol type, habitual consumption levels and sex differences. Heavy and regular alcohol consumption has been positively correlated with increasing body weight, although it is unclear whether this is due to alcohol consumption per se or to additional energy intake from food. This review explores the effects of alcohol consumption on food energy intake in healthy adults. CINAHL Plus, EMBASE, Medline and PsycINFO were searched through February 2018 for crossover and randomised controlled trials where an alcohol dose was compared with a non-alcohol condition. Study quality was assessed using the Effective Public Health Practice Project tool. A total of twenty-two studies involving 701 participants were included from the 18427 papers retrieved. Studies consistently demonstrated no compensation for alcoholic beverage energy intake, with dietary energy intake not decreasing due to alcoholic beverage ingestion. Meta-analyses using the random-effects model were conducted on twelve studies and demonstrated that alcoholic beverage consumption significantly increased food energy intake and total energy intake compared with a non-alcoholic comparator by weighted mean differences of 343 (95\% CI 161, 525) and 1072 (95\% CI 820, 1323) kJ, respectively. Generalisability is limited to younger adults (18-37 years), and meta-analyses for some outcomes had substantial statistical heterogeneity or evidence of small-study effects. This review suggests that adults do not compensate appropriately for alcohol energy by eating less, and a relatively modest alcohol dose may lead to an increase in food consumption.

\section{Key words: Alcohol consumption: Food energy intake: Total energy intake: Food intake: Systematic reviews}

In 2014, the WHO reported that $38 \%$ of men and $40 \%$ of women were classified as overweight and $11 \%$ of men and $15 \%$ of women were classified as obese, with prevalence of both trending upwards ${ }^{(1)}$. Total alcohol per capita consumption also increased from 2005 to 2010 and predicted an upward trajectory $^{(2)}$. With an energy density of $29 \mathrm{~kJ} / \mathrm{g}$, second only to dietary fat, it is logical to hypothesise that in those who voluntarily consume alcoholic beverages, this will contribute to their overall energy intake ${ }^{(3)}$. However, the relationship between alcoholic beverage consumption and overweight and obesity is complex and inconsistent ${ }^{(3)}$. Alcohol type, habitual consumption patterns and sex are factors that may mediate the relationship between alcohol consumption and body weight. Evidence suggests that type of alcoholic beverage is important; for example, wine has been shown to be more protective against weight gain than beer and spirits in observational studies $^{(4)}$. In women, mild to moderate alcohol consumption is associated with lower weight, whereas in men, moderate intake was associated with higher weight ${ }^{(4)}$. Bendsen et al $^{(5)}$ reviewed thirty-five observational studies on the association between beer consumption and general obesity. A positive association or no association between beer consumption and general obesity was observed in men, whereas an inverse association or no association with general obesity was observed in women ${ }^{(5)}$. Analyses conducted on the 2008-2014 Healthy Survey for England and the Scottish Health Survey found that in both male and female young adults, those consuming the highest levels of alcohol on a single drinking session were more likely to be overweight or obese compared with those with the lowest intake ${ }^{(6)}$.

Aside from alcohol type and sex differences, it has been suggested a dose-dependent response exists as 'heavy' alcohol drinking, defined as greater than two to three standard drinks per d, was more positively associated with increased body weight compared with low to moderate alcohol drinking ${ }^{(4)}$. Across all WHO regions in 2014, the prevalence of heavy episodic drinking in people aged 15 years and over was 12.3 and $2.9 \%$ for males and females, respectively ${ }^{(2)}$. To add further complexity, a J-shaped association between alcohol consumption and negative health outcomes has been reported, with low

Abbreviations: E-beverage, energy-containing beverages; N-beverage, no beverage; NE-beverage, beverages that contained no or negligible energy.

* Corresponding author: A. Kwok, fax +6139902 4278, email alastair.kwok@monash.edu 
to moderate alcohol consumption correlated with reduced risk of multiple cardiovascular outcomes; whereas high alcohol consumption is correlated with increased risk of oral, pharyngeal and oesophageal cancers ${ }^{(7,8)}$.

Alcohol consumption has been suggested to stimulate appetite and potentially increase food intake. Although the mechanisms are unclear, it has been postulated that ingestion of alcohol appears to bypass the satiety mechanisms that modulate short term food intake ${ }^{(9)}$. Alcohol has been proposed to support an overall increase in food intake in two different pathways: (1) binding to type-A gamma-aminobutyric acid $\left(\mathrm{GABA}_{\mathrm{A}}\right)$ receptors and stimulating the release of opioid and (2) decreasing the serotonin response, a hunger suppresser ${ }^{(3,9)}$. Alcoholic beverages may contribute to passive overconsumption of energy from foods. The relatively high energy density of alcoholic beverages may be additive to food energy intake, meaning it may be easier to unintentionally consume excess dietary energy ${ }^{(9,10)}$.

Studies consistently demonstrate that energy gained through the consumption of alcoholic beverages is not compensated for by eating less food in the short-term ${ }^{(10-12)}$. Energy compensation involves the modification of energy intake in response to previous consumption of either food or beverage and is fundamental for regulating energy balance ${ }^{(13,14)}$. Energy compensation in response to consumption of beverages is generally poor, particularly when compared with energy compensation in response to consumption of semi-solid or solid food ${ }^{(13)}$. A combination of increased appetite and poor compensation of energy from alcohol could contribute to the impact on energy intake in regular consumers of alcoholic beverages.

The effects of alcohol consumption on food intake have been reviewed before $2012^{(9,10,15-17)}$. Three narrative reviews ${ }^{(9,10,15)}$ suggested that alcohol consumption increases acute food intake, and a meta-analysis ${ }^{(16)}$ concluded that alcohol consumption increased the total energy intake. A minireview ${ }^{(17)}$ with limited scope further supported that alcohol consumption increased food energy intake, however, only at high alcohol doses and cautioned the findings due to the small sample sizes of the included studies ${ }^{(18)}$. No extensive systematic review with meta-analysis has been previously conducted to investigate the effects of alcohol consumption on both food energy intake and total energy intake in adult humans.

Hence, the aim of this review was to determine the impact of alcohol consumption on (1) food energy intake and (2) total energy intake (the sum of both the beverage and food consumption). This review aimed to elicit a stronger understanding of whether there is a dose response to alcohol consumption that impacts energy intake or whether other factors, such as the energy content of the comparator beverage, is important.

\section{Methods}

A systematic review with meta-analysis of aggregate data was reported in accordance with the Preferred Reporting Items for Systematic Reviews and Meta-Analyses guidelines ${ }^{(19)}$. A review protocol does not exist.
Table 1. Participant, intervention, comparator, outcome, study design, inclusion and exclusion criteria used to determine study eligibility

\begin{tabular}{|c|c|c|}
\hline & Inclusion criteria & Exclusion criteria \\
\hline Population & Healthy populations & $\begin{array}{l}\text { Populations with acute or } \\
\text { chronic diseases }\end{array}$ \\
\hline Intervention & $\begin{array}{l}\text { Consumption of an alcoholic } \\
\text { beverage with a specified } \\
\text { alcohol dose }\end{array}$ & $\begin{array}{l}\text { Consumption of ad libitum } \\
\text { alcoholic beverage or } \\
\text { alcohol mixed into a meal }\end{array}$ \\
\hline Comparator & $\begin{array}{l}\text { Consumption of a non- } \\
\text { alcoholic beverage or } \\
\text { no beverage }\end{array}$ & Not applicable \\
\hline Outcomes & $\begin{array}{l}\text { Report mean differences in } \\
\text { food intake, food energy } \\
\text { intake or total energy } \\
\text { intake, with or after } \\
\text { consumption of a } \\
\text { beverage or no provided } \\
\text { beverage }\end{array}$ & $\begin{array}{l}\text { Studies that do not report a } \\
\text { mean difference in food } \\
\text { intake, food energy intake } \\
\text { or total energy intake, with } \\
\text { or after consumption of a } \\
\text { beverage or no provided } \\
\text { beverage }\end{array}$ \\
\hline Study design & $\begin{array}{l}\text { Randomised controlled trial, } \\
\text { randomised crossover } \\
\text { and crossover trials }\end{array}$ & All other study designs \\
\hline
\end{tabular}

\section{Eligibility criteria}

The participant, intervention, comparator, outcome, study design was used to develop eligibility criteria for study inclusion (Table 1). Original peer-reviewed randomised controlled trials (RCT), randomised crossover or non-randomised crossover trials only were eligible for inclusion.

\section{Search strategy}

A systematic search was conducted for studies comparing alcohol consumption against a non-alcoholic comparator on ad libitum food energy intake in healthy adult participants ( $\geq 18$ years of age) with no date limits and all papers up to 1 st February 2018 were included. A database search was conducted of CINAHL Plus, Embase, Medline and PsycINFO by one author (A. K.). A proximal search strategy was employed, linking the terms or variations of 'alcohol drinking', 'alcoholic beverages', 'beer', 'wine', 'spirits', 'food intake', 'energy intake', 'eating behaviour', 'appetite' and 'satiety'. Table 2 provides the Ovid Medline search strategy. Limits were applied to exclude non-English-language articles and animal studies.

\section{Literature screening}

All retrieved articles were independently assessed for inclusion by two authors (A. K. and G. P.) after duplicates were removed electronically. Endnote X7 (Clarivate Analytics) was used to store all the references, and Covidence (Covidence) was used to screen articles for eligibility. Studies were excluded on the basis of title and abstract. Full-text articles were then obtained and assessed for eligibility independently by two authors. A third author (H. T.) was consulted for any discrepancies.

\section{Data extraction}

Data extraction for each eligible study was conducted independently by two authors (A. K. and G. P.) using a specifically 
Table 2. Full search strategy for Ovid Medline

1. Alcohol drinking/ or alcohol drinking in college/

2. Alcoholic beverages/ or absinthe/ or beer/ or wine/

3. ((alcohol* adj3 (drink* or intake* or consum $\left.{ }^{*}\right)$ ) or alcohol* beverage $^{*}$ or beer $^{\star}$ or wine ${ }^{\star}$ or spirits or liquor ${ }^{\star}$ or liquer ${ }^{\star}$ or liqueur ${ }^{\star}$ or aperitif*).ti,ab.

4. Eating/

5. Energy Intake/

6. Appetite/or Appetite Regulation/

7. Satiation/or Satiety Response/

8. Meals/

9. Feeding Behavior/

10. ((food adj3 (intake or consum $\left.{ }^{\star}\right)$ ) or (energy adj3 (intake or consum*)) or appetite or satiety or satiat ${ }^{*}$ or eat* ${ }^{*}$ or snack ${ }^{*}$ or meal ${ }^{*}$ or calori ${ }^{*}$ intake or feeding behavio?r).ti,ab.

11. 1 or 2 or 3

12. 4 or 5 or 6 or 7 or 8 or 9 or 10

13. 11 AND 12

14. Humans/ and Animals/

15. Animals/ not 14

16. 13 not 15

17. Limit 16 to English language

developed standardised data extraction tool, which included publication details, study design, study procedures and classification of hierarchy of evidence according to the National Health and Medical Research Council guidelines ${ }^{(20)}$. Sample size and participant characteristics were extracted including age, sex, baseline BMI, usual alcohol consumption and degree of restrained eating. Specific to alcohol, details of the dose, type of alcoholic beverage, volume and the non-alcoholic comparator were recorded. Non-alcoholic comparators used in the included studies were coded into three groups: (1) beverages that contained no or negligible energy (NE-beverage), (2) no beverage (N-beverage) and (3) energy-containing beverages (E-beverage). The timing of the alcohol and comparator beverage provision relative to the consumption of the food was recorded along with compliance measures, time to follow-up, potential study bias and funding sources. Outcome measures for both the alcoholic and non-alcoholic beverage comparators included differences in food energy intake (kJ), total energy intake $(\mathrm{kJ})$ or food mass $(\mathrm{g})$ consumed. Total energy intake was defined as the sum of food energy intake and beverage energy intake. Body weight change was also recorded where reported. For each measure, the mean and either the standard deviation or the standard error of the mean were recorded where reported as well as corresponding $P$ values between the intervention and comparator groups. All data extraction were completed on Microsoft Excel 2013 (Microsoft). Any disparities in the data extracted were discussed between the two authors (A. K. and G. P.). A third author (H. T.) was consulted with unresolved discrepancies. Authors were contacted in an attempt to retrieve missing data. A total of twenty-two requests for data were sent to authors, with a response rate of $45 \%$ (ten requests). However, of the ten respondents, four did not have access or no longer held these data.

\section{Risk of bias and quality}

Study quality and risk of bias were independently assessed by two authors (A. K. and G. P.) using the Effective Public Health Practice Project (EPHPP) quality assessment tool for quantitative studies ${ }^{(21)}$. The EPHPP quality assessment tool for quantitative studies was chosen as this systematic review included non-randomised quantitative studies. Each paper was rated as strong, moderate or weak. Any discrepancies were dealt with by consensus discussion with the third author (H. T.).

\section{Statistical analysis}

To analyse the impact of alcohol dose on food energy intake via meta-analyses, the alcohol dose from the alcoholic beverage intervention was categorised into either low $(<30 \mathrm{~g}$ or $<0.6 \mathrm{~g} / \mathrm{kg}$ ) or high dose $(\geq 30 \mathrm{~g}$ or $\geq 0.6 \mathrm{~g} / \mathrm{kg})$. As different countries have different guidelines for low-risk drinking, a fixed alcohol dose of $30 \mathrm{~g}$ was used to define low and high alcohol doses based on the Australian National Guidelines for Alcohol Consumption $^{(22,23)}$. An alcohol dose of $30 \mathrm{~g}$ is the midpoint between low-risk drinking per $\mathrm{d}(20 \mathrm{~g})$ and the maximum dose to reduce the risk of alcohol-related injury from a single occasion ( $40 \mathrm{~g}$ ) in both males and females ${ }^{(23)}$. An adjusted alcohol dose of $0.6 \mathrm{~g} / \mathrm{kg}$ of body weight was considered as a priming dose for increasing alcohol consumption and potentially increasing food consumption ${ }^{(24,25)}$

We conducted combined analyses of food energy intake and total energy intake, where reported or provided by authors, and sub-group analyses of comparator type (NE-beverage, $\mathrm{N}$-beverage and E-beverage comparators) and alcohol dose. Groups were combined for pairwise comparisons where required. The mean difference and standard error of the mean difference from each study was used to analyse data. For randomised crossover trials and non-randomised crossover trials, a correlation coefficient of 0.5 was used to impute the standard error of the mean difference in food energy intake and total energy intake, if not reported in the publication or provided by the authors. Sensitivity analysis was conducted using correlation coefficients of $0 \cdot 2$ and $0 \cdot 8$. Heterogeneity was assessed using the $Q$ statistic, and its $P$ value and inconsistency were assessed using an extension of the $Q$ statistic, the $I^{2}$ statistic. Values of $P<0.10$ or values of $I^{2}>50 \%$ suggested substantial heterogeneity. Given the diversity in clinical characteristics across studies, and our aim to estimate the average (rather than common) effect across studies, we used a randomeffects model in all meta-analyses and sub-group analyses. For our primary analyses, we used the between-study variance estimator developed by DerSimonian \& Laird ${ }^{(26)}$ and calculated the Wald-type normal distribution CI. We also performed a sensitivity analysis using the Hartung-Knapp-Sidik-Jonkman (HKSJ) method for random-effects meta-analyses ${ }^{(27)}$. Both a qualitative (funnel plot) and a quantitative (Egger's regression 
test) approach were used to examine the potential small-study effects, the tendency for smaller studies to have systematically different results when compared with larger studies. An influence analysis was conducted using the random-effects model (DerSimonian and Laird) to determine the effect of removing each included study on the overall effect and 95\% CI. The meta-analyses, funnel plot and Egger's regression test were conducted using Stata 13 software (Stata Corp.) with the metan, metafunnel and metabias packages. The influence analysis was conducted using MetaXL 5.3 (Epigear International). A $P$ value of $<0.05$ for meta-analyses was considered statistically significant.

\section{Results}

\section{Study selection and characteristics}

A total of 18427 papers were retrieved (Ovid Medline $=5509$, EMBASE $=9480$, CINAHL Plus $=2259$, PsycINFO =1179). One paper $^{(28)}$ reported two different studies of which both were eligible, whereas two papers ${ }^{(29,30)}$ reported two different studies with only one being eligible in each paper. In total, twenty-two separate studies met the PICOS inclusion criteria and were included (Fig. 1). The study characteristics for the twenty-two studies are provided (Table 3). Nine studies ${ }^{(30-38)}$ were excluded and justification for exclusion is provided in online Supplementary Table S1.

\section{Study design}

Thirteen studies ${ }^{(12,28,29,39-41,45,47,49-51-54)}$ employed a randomised crossover trial design, four utilised ${ }^{(24,42,46,48)}$ a randomised parallel trial design and five, a non-randomised crossover trial design ${ }^{(11,43,44,52,53)}$

Sixteen studies ${ }^{12,24,28,39,40,42,45-49,51-54)}$ utilised a pre-load paradigm design where ingestion of a beverage occurred a set period of time before the consumption of food. The time between consuming the pre-load and consuming the first test food varied between studies, from $10 \mathrm{~min}^{(46)}$ up to $2 \mathrm{~h}^{(28)}$. In all of these studies, alcohol ingestion was compared with nonalcoholic comparator conditions. Six studies ${ }^{(12,29,39-41,45)}$ provided a standardised control meal for participants before the start of the study, five $\mathrm{e}^{(28,49,52-54)}$ instructed participants to consume their own 'usual' meal and one study ${ }^{(28)}$ instructed participants to fast before the testing.
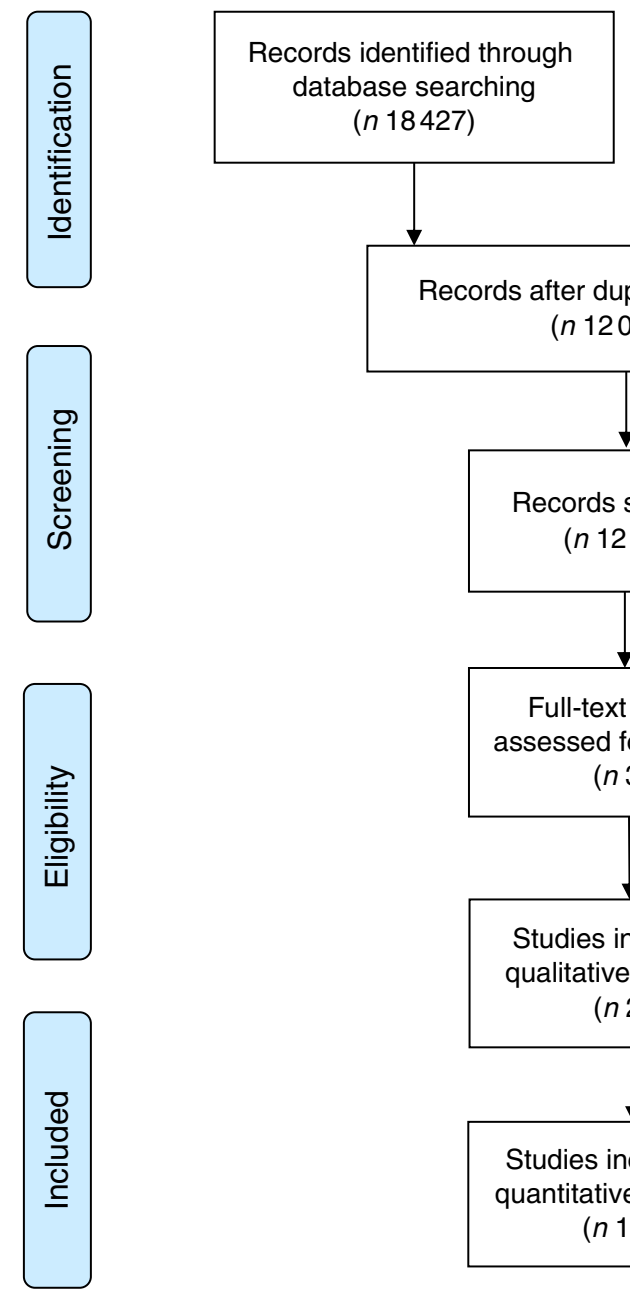

\section{Additional records identified through other sources} ( $n 0)$

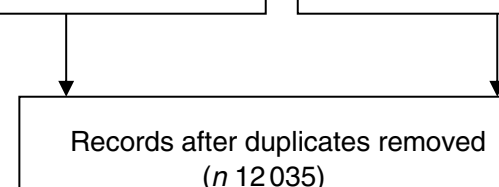

Records excluded based on titles and abstracts (n 12005)

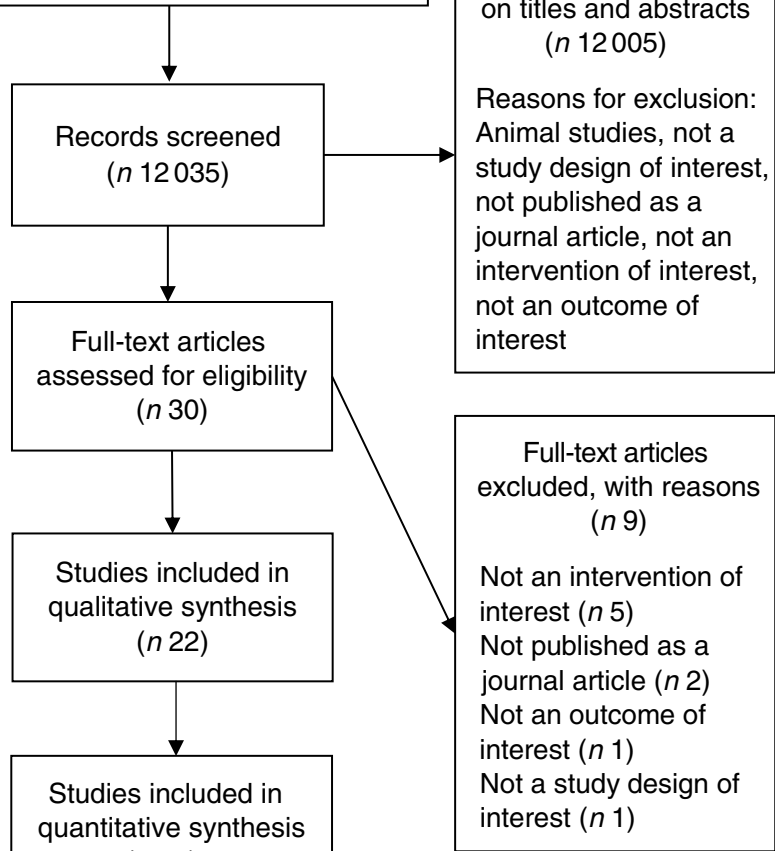

(n 12)

Fig. 1. Study selection flow diagram. 


\section{Nes British Journal of Nutrition}

Table 3. Study characteristics for the twenty-two trials meeting the full inclusion criteria

\begin{tabular}{|c|c|c|c|c|c|c|c|c|c|}
\hline $\begin{array}{l}\text { Reference and } \\
\text { country }\end{array}$ & Study design & Subjects $(n)$ and sex & Age (years) and BMl $\left(\mathrm{kg} / \mathrm{m}^{2}\right)$ & $\begin{array}{l}\text { Timing of } \\
\text { beverage } \\
\text { consumption }\end{array}$ & Intervention & Volume (ml) & Alcohol dose (g) & Comparator & Volume (ml) \\
\hline $\begin{array}{l}\text { Buemann et al. } \\
(2002)^{(29)}, \\
\text { Denmark }\end{array}$ & $\begin{array}{l}\text { Three-arm, } \\
\text { randomised } \\
\text { crossover trial }\end{array}$ & 22 males & $\begin{array}{l}\text { Aged } 20 \cdot 0-33 \cdot 0 \\
\text { BMI range between } 19.9 \text { and } 25.5\end{array}$ & $\begin{array}{l}\text { Before and with } \\
\text { the food }\end{array}$ & $\begin{array}{l}\text { Red wine } \\
\text { Lager beer }\end{array}$ & $\begin{array}{l}3.2 \mathrm{ml} / \mathrm{kg}^{*} \\
9.0 \mathrm{ml} / \mathrm{kg}^{\star}\end{array}$ & $\begin{array}{l}0.3 \mathrm{~g} / \mathrm{kg} \dagger \\
0.3 \mathrm{~g} / \mathrm{kg}+\end{array}$ & Carbonated soft drink & $9.0 \mathrm{ml} / \mathrm{kg}^{*}$ \\
\hline $\begin{array}{l}\text { Caton et al. } \\
(2004)^{(39)} \text {, UK }\end{array}$ & $\begin{array}{l}\text { Single-blind, } \\
\text { three-arm, } \\
\text { randomised } \\
\text { crossover trial }\end{array}$ & 12 males & $\begin{array}{l}\text { Aged } 18 \cdot 0-35 \cdot 0 \\
\mathrm{BMI}<26.0 \\
\text { Excluded restrained eaters }\end{array}$ & $\begin{array}{l}30 \text { min before } \\
\text { food was } \\
\text { served }\end{array}$ & $\begin{array}{l}\text { Lager }(32 \mathrm{~g} \text { alcohol) } \\
\text { Lager }(8 \mathrm{~g} \text { alcohol })\end{array}$ & $\begin{array}{l}370 \\
340\end{array}$ & $\begin{array}{l}32 \cdot 0 \\
8.0\end{array}$ & No alcohol lager & 330 \\
\hline \multirow[t]{2}{*}{$\begin{array}{l}\text { Caton et al. } \\
\quad(2005)^{(40)}, \text { UK }\end{array}$} & \multirow{2}{*}{$\begin{array}{l}\text { Single-blind, } \\
\text { four-arm, } \\
\text { randomised } \\
\text { crossover trial }\end{array}$} & \multirow[t]{2}{*}{12 males } & $\begin{array}{l}\text { Aged } 18 \cdot 0-50 \cdot 0 \\
\mathrm{BMI}<27 \cdot 0\end{array}$ & \multirow{2}{*}{$\begin{array}{l}20 \text { min before } \\
\text { food was } \\
\text { served }\end{array}$} & \multirow{2}{*}{$\begin{array}{l}\text { Carbonated grape juice } \\
\text { with alcohol and bland } \\
\text { foods } \\
\text { Carbonated grape juice } \\
\text { with alcohol and } \\
\text { flavoured foods }\end{array}$} & 405 & $24 \cdot 0$ & $\begin{array}{l}\text { Carbonated grape juice } \\
\text { and bland foods }\end{array}$ & 405 \\
\hline & & & Excluded restrained eaters & & & 405 & 24.0 & $\begin{array}{l}\text { Carbonated grape juice } \\
\text { and flavoured foods }\end{array}$ & 405 \\
\hline $\begin{array}{l}\text { Caton et al. } \\
\quad(2007)^{(41)}, \text { UK }\end{array}$ & $\begin{array}{l}\text { Three-arm, } \\
\text { randomised } \\
\text { crossover trial }\end{array}$ & 12 males & $\begin{array}{l}\text { Mean age } 24.7 \text { (SD 1.9) } \\
\text { Mean BMI } 24 \text { (SD 0.4) } \\
\text { Excluded restrained eaters }\end{array}$ & $\begin{array}{l}\text { Either before or } \\
\text { during the } \\
\text { consumption } \\
\text { of the food }\end{array}$ & $\begin{array}{l}\text { Red wine (aperitif) } \\
\text { Red wine (co-ingestion) }\end{array}$ & $\begin{array}{l}375 \\
375\end{array}$ & $\begin{array}{l}38 \cdot 5 \\
38 \cdot 5\end{array}$ & No beverage & 0 \\
\hline $\begin{array}{l}\text { Christiansen } \\
\text { et al. } \\
(2016)^{(42)} \text {, UK }\end{array}$ & $\begin{array}{l}\text { Single blind, } \\
\text { two-arm, RCT }\end{array}$ & 60 females & $\begin{array}{l}\text { Mean age } 19.6(\mathrm{sD} 4.0) \\
\text { Mean BMI } 20.8(\mathrm{sD} 2.8)\end{array}$ & $\begin{array}{l}10 \text { min before } \\
\text { food was } \\
\text { served }\end{array}$ & Vodka and diet lemonade & NR & $0.6 \mathrm{~g} / \mathrm{kg} \dagger$ & Diet lemonade & NR \\
\hline $\begin{array}{l}\text { Cordain et al. } \\
\quad(1997)^{(43)} \\
\text { USA }\end{array}$ & $\begin{array}{l}\text { Two-arm, } \\
\text { crossover trial }\end{array}$ & 14 males & $\begin{array}{l}\text { Mean age } 32 \cdot 1 \text { (SEM 2.4) } \\
\text { BMl: NR }\end{array}$ & $\begin{array}{l}\text { Co-administered } \\
\text { with the } \\
\text { evening meal } \\
\text { daily }\end{array}$ & $\begin{array}{l}\text { Red wine (daily for } \\
6 \text { weeks) }\end{array}$ & 270 & $27 \cdot 6$ & $\begin{array}{l}\text { Abstinence from } \\
\text { alcoholic beverages } \\
\text { for } 6 \text { weeks }\end{array}$ & NR \\
\hline $\begin{array}{l}\text { Cordain et al. } \\
\quad(2000)^{(44)} \\
\text { USA }\end{array}$ & $\begin{array}{l}\text { Two-arm, } \\
\text { crossover trial }\end{array}$ & 20 females & $\begin{array}{l}\text { Aged 30.0-50.0 } \\
\text { Mean BMI 29.8 (SEM 2.2) }\end{array}$ & $\begin{array}{l}\text { Co-administered } \\
\text { with the } \\
\text { evening meal } \\
\text { on } 5 \mathrm{~d} \text { each } \\
\text { week }\end{array}$ & $\begin{array}{l}\text { Red wine (each day, for } \\
5 \mathrm{~d} \text { each week for } \\
10 \text { weeks) }\end{array}$ & 270 & 19.4 & $\begin{array}{l}\text { Abstinence from any } \\
\text { alcoholic beverages } \\
\text { for } 10 \text { weeks }\end{array}$ & NR \\
\hline \multirow{2}{*}{$\begin{array}{l}\text { Foltin et al. } \\
\quad(1993)^{(11)} \\
\quad \text { USA }\end{array}$} & \multirow{2}{*}{$\begin{array}{l}\text { Single-blind, } \\
\text { five-arm, } \\
\text { parallel } \\
\text { crossover trial }\end{array}$} & \multirow[t]{2}{*}{6 males } & $\begin{array}{l}\text { Mean age } 28 \cdot 2 \text { (SEM 2.2) } \\
\text { BMI: NR }\end{array}$ & \multirow{2}{*}{$\begin{array}{c}\text { Beverages were } \\
\text { consumed at } \\
09.30,13.15 \\
16.45 \text { and } \\
20.15 \text { hours }\end{array}$} & $\begin{array}{l}\text { Low-energy cocktail of } \\
\text { juice and alcohol }\end{array}$ & 300 & $0.3 \mathrm{~g} / \mathrm{kg} \dagger$ & $\begin{array}{l}\text { Low-energy cocktail of } \\
\text { juice and dextrose }\end{array}$ & 300 \\
\hline & & & Excluded restrained eaters & & $\begin{array}{l}\text { High-energy cocktail of } \\
\text { juice and alcohol }\end{array}$ & 300 & $0.5 \mathrm{~g} / \mathrm{kg} \dagger$ & $\begin{array}{l}\text { High-energy cocktail of } \\
\text { juice and dextrose } \\
\text { No beverage }\end{array}$ & 300 \\
\hline $\begin{array}{l}\text { Hetherington } \\
\quad \text { et al. } \\
(2001)^{(45)}, \text { UK }\end{array}$ & $\begin{array}{l}\text { Single blind, } \\
\text { three arm, } \\
\text { randomised } \\
\text { crossover trial }\end{array}$ & 26 males & $\begin{array}{l}\text { Aged } 18 \cdot 0-40 \cdot 0 \\
\text { BMl } 20 \cdot 0-27 \cdot 0 \\
\text { Excluded restrained eaters }\end{array}$ & $\begin{array}{l}30 \text { min before } \\
\text { food was } \\
\text { served }\end{array}$ & Lager & 330 & $24 \cdot 0$ & $\begin{array}{l}\text { No-alcohol lager } \\
\text { No beverage }\end{array}$ & $\begin{array}{c}330 \\
0\end{array}$ \\
\hline $\begin{array}{l}\text { Hofmann et al. } \\
(2008)^{(46)} \\
\text { Germany }\end{array}$ & $\begin{array}{l}\text { Single-blind, } \\
\text { two-arm, RCT }\end{array}$ & 63 females & $\begin{array}{l}\text { Mean age } 21.6(\mathrm{sD} 2.4) \\
\text { Mean BMI 21.8 (SD 2.2) }\end{array}$ & $\begin{array}{l}10 \text { min before } \\
\text { food was } \\
\text { served }\end{array}$ & Vodka and OJ & 300 & $0.4 \mathrm{~g} / \mathrm{kg} \dagger$ & OJ & 300 \\
\hline \multirow{3}{*}{$\begin{array}{l}\text { Hollister } \\
\quad(1970)^{(28)} \\
\text { USA }\end{array}$} & \multirow{3}{*}{$\begin{array}{l}\text { Study 1: double- } \\
\text { blind, four-arm, } \\
\text { randomised } \\
\text { crossover trial }\end{array}$} & \multirow[t]{3}{*}{$\begin{array}{l}12 \text { (eleven males, one } \\
\text { female) }\end{array}$} & $\begin{array}{l}\text { Age NR } \\
\text { BMI NR }\end{array}$ & \multirow{3}{*}{$\begin{array}{l}\text { Before the } \\
\text { consumption } \\
\text { of the } \\
\text { milkshakes, } \\
\text { up to } 5 \mathrm{~h} \\
\text { beforehand }\end{array}$} & \multirow[t]{3}{*}{$\begin{array}{l}\text { Diet soft drink with } \\
\text { alcohol }\end{array}$} & \multirow[t]{3}{*}{180} & \multirow[t]{3}{*}{$0.8 \mathrm{~g} / \mathrm{kg} \dagger$} & $\begin{array}{l}\text { Diet soft drink with } \\
\text { marijuana }\end{array}$ & 180 \\
\hline & & & & & & & & $\begin{array}{l}\text { Diet soft drink with } \\
\text { dextroamphetamine } \\
\text { sulphate }\end{array}$ & 180 \\
\hline & & & & & & & & $\begin{array}{l}\text { Diet soft drink with } \\
\text { marijuana, } \\
\text { cannaboids removed }\end{array}$ & 180 \\
\hline \multirow{2}{*}{$\begin{array}{l}\text { Hollister } \\
\quad(1970)^{(28)} \\
\quad \text { USA }\end{array}$} & \multirow{2}{*}{$\begin{array}{l}\text { Study 2: double- } \\
\text { blind, three- } \\
\text { arm, } \\
\text { randomised } \\
\text { crossover trial }\end{array}$} & \multirow[t]{2}{*}{12 males } & Age NR & \multirow{3}{*}{$\begin{array}{l}\text { Before the } \\
\text { consumption } \\
\text { of the } \\
\text { milkshakes, } \\
\text { up to } 5 \mathrm{~h} \\
\text { beforehand } \\
\text { Co-administered } \\
\text { with a } \\
\text { controlled } \\
\text { snack on } \\
\text { day } 2\end{array}$} & \multirow[t]{2}{*}{$\begin{array}{l}\text { Diet soft drink with } \\
\text { alcohol }\end{array}$} & \multirow[t]{2}{*}{180} & \multirow[t]{2}{*}{$0.5 \mathrm{~g} / \mathrm{kg} \dagger$} & $\begin{array}{l}\text { Diet soft drink with } \\
\text { marijuana }\end{array}$ & 180 \\
\hline & & & BIMII NR & & & & & $\begin{array}{l}\text { Diet soft drink with } \\
\text { dextroamphetamine } \\
\text { sulphate }\end{array}$ & 180 \\
\hline $\begin{array}{l}\text { Mattes } \\
\quad(1996)^{(47)} \\
\quad \text { USA }\end{array}$ & $\begin{array}{l}\text { Five-arm } \\
\quad \text { randomised } \\
\text { crossover trial }\end{array}$ & $\begin{array}{l}16 \text { (eight males, eight } \\
\text { females) }\end{array}$ & $\begin{array}{l}\text { Males } \\
\text { Mean age } 27.1 \text { (sD 6.2) } \\
\text { Mean BMI 27.6 (SD } 4.8) \\
\text { Females } \\
\text { Mean age 23.2 (sD 2.0) } \\
\text { Mean BMI 21.2 (sD 3.1) } \\
\text { Excluded restrained eaters }\end{array}$ & & $\begin{array}{l}\text { Beer }(5 \% \text { alcohol }) \\
\text { Beer }(2.9 \% \text { alcohol }) \\
\text { Beer }(0.1 \% \text { alcohol })\end{array}$ & $\begin{array}{l}1080 \\
1080 \\
1080\end{array}$ & $\begin{array}{l}54.0 \\
31.3 \\
10.8\end{array}$ & $\begin{array}{l}\text { Cola } \\
\text { Carbonated water }\end{array}$ & $\begin{array}{l}1080 \\
1080\end{array}$ \\
\hline
\end{tabular}




\section{N British Journal of Nutrition}

\begin{tabular}{|c|c|c|c|c|c|c|c|c|c|}
\hline $\begin{array}{l}\text { Reference and } \\
\text { country }\end{array}$ & Study design & Subjects $(n)$ and sex & Age (years) and BMI $\left(\mathrm{kg} / \mathrm{m}^{2}\right)$ & $\begin{array}{l}\text { Timing of } \\
\text { beverage } \\
\text { consumption }\end{array}$ & Intervention & Volume (ml) & Alcohol dose (g) & Comparator & Volume (ml) \\
\hline $\begin{array}{l}\text { Ouwens et al. } \\
(2003)^{(48)} \text {, } \\
\text { The } \\
\text { Netherlands }\end{array}$ & $\begin{array}{l}\text { Single blind, } \\
\text { two-arm, RCT }\end{array}$ & 119 females & $\begin{array}{l}\text { Mean age } 21.1 \text { (sD 2.3) } \\
\text { Mean BMI 23.1 (SD 2.9) }\end{array}$ & $\begin{array}{l}20 \text { min before } \\
\text { the food was } \\
\text { served }\end{array}$ & Vodka and OJ & 400 & 24.4 & OJ & 400 \\
\hline $\begin{array}{l}\text { Poppitt et al. } \\
\quad(1996)^{(12)}, \text { UK }\end{array}$ & $\begin{array}{l}\text { Single blind, } \\
\text { four-arm } \\
\text { randomised } \\
\text { crossover trial }\end{array}$ & 20 females & $\begin{array}{l}\text { Mean age } 37.0 \text { (SD 11.4) } \\
\text { Mean BMI 23.0 (sD 2.8) }\end{array}$ & $\begin{array}{l}30 \text { min before } \\
\text { the food was } \\
\text { served }\end{array}$ & Gin and tonic & $392 \mathrm{~g} \neq$ & 30.6 & $\begin{array}{l}\text { Gin-flavoured slimline } \\
\text { tonic and water } \\
\text { Gin-flavoured slimline } \\
\text { tonic, water and } \\
\text { maltodextrin }\end{array}$ & $\begin{array}{l}392 \mathrm{gf} \\
392 \mathrm{gf}\end{array}$ \\
\hline \multirow[t]{2}{*}{$\begin{array}{l}\text { Rose et al. } \\
\qquad(2015)^{(24)} \text {, UK }\end{array}$} & \multirow{2}{*}{$\begin{array}{l}\text { Four-arm, } \\
\text { randomised } \\
\text { controlled } \\
\text { parallel trial }\end{array}$} & \multirow[t]{2}{*}{$\begin{array}{l}114 \text { (sixty-six females, } \\
\text { forty-eight males) }\end{array}$} & $\begin{array}{l}\text { Vodka and bar-lab } \\
\text { Mean age } 20.5 \text { (sD 3.2) } \\
\text { Mean BMI NR }\end{array}$ & \multirow[t]{2}{*}{$\begin{array}{l}20 \text { min before } \\
\text { food was } \\
\text { served }\end{array}$} & $\begin{array}{l}\text { Vodka and diet lemonade } \\
\text { and bar-lab } \\
\text { environment }\end{array}$ & 400 & $0.6 \mathrm{~g} / \mathrm{kg} \dagger$ & $\begin{array}{l}\text { Diet soft drink and bar- } \\
\text { lab environment }\end{array}$ & 400 \\
\hline & & & $\begin{array}{l}\text { Vodka and sterile lab } \\
\text { Mean age 19.8 (sD 1.4) } \\
\text { Mean BMI NR } \\
\text { Soft drink and bar-lab } \\
\text { Mean age 20.0 (sD 2.7) } \\
\text { Mean BMI NR } \\
\text { Soft drink and sterile lab } \\
\text { Mean age } 20.7 \text { (sD 1.5) } \\
\text { Mean BMI NR }\end{array}$ & & $\begin{array}{l}\text { Vodka and diet lemonade } \\
\text { and sterile lab } \\
\text { environment }\end{array}$ & 400 & $0.6 \mathrm{~g} / \mathrm{kg} \dagger$ & $\begin{array}{l}\text { Diet soft drink and sterile } \\
\text { lab environment }\end{array}$ & 400 \\
\hline \multirow{2}{*}{$\begin{array}{l}\text { Schrieks et al. } \\
(2015)^{(49)} \text {, } \\
\text { The } \\
\text { Netherlands }\end{array}$} & \multirow{2}{*}{$\begin{array}{l}\text { Single-blind, } \\
\text { six-arm, } \\
\text { randomised } \\
\text { crossover trial }\end{array}$} & \multirow[t]{2}{*}{24 males } & $\begin{array}{l}\text { Mean age } 32.0 \text { (SD } 0.8) \\
\text { Mean BMI } 23.0(\text { SD } 0.1) \\
\text { Excluded restrained eaters }\end{array}$ & \multirow[t]{2}{*}{$\begin{array}{l}45 \text { min before } \\
\text { the food was } \\
\text { served }\end{array}$} & $\begin{array}{l}\text { Vodka and OJ with } 40 \mathrm{~g} \\
\text { butter cake } \\
\text { consumption }\end{array}$ & 200 & $20 \cdot 0$ & $\begin{array}{l}\text { OJ with maltodextrin and } \\
40 \mathrm{~g} \text { butter cake } \\
\text { consumption }\end{array}$ & 200 \\
\hline & & & Excluded restrained eaters & & $\begin{array}{l}\text { Vodka and OJ with } 40 \mathrm{~g} \\
\text { cake mock sham } \\
\text { feeding }\end{array}$ & 200 & $20 \cdot 0$ & $\begin{array}{l}\text { OJ with maltodextrin with } \\
40 \mathrm{~g} \text { cake mock sham } \\
\text { feeding }\end{array}$ & 200 \\
\hline \multirow{2}{*}{$\begin{array}{l}\text { Tremblay et al. } \\
\text { (1995)(50), } \\
\text { Canada }\end{array}$} & \multirow{2}{*}{$\begin{array}{l}\text { Single blind, } \\
\quad \text { four-arm, } \\
\text { randomised } \\
\text { crossover trial }\end{array}$} & \multirow[t]{2}{*}{8 males } & $\begin{array}{l}\text { Mean age } 36.4(\mathrm{sD} 6.9) \\
\text { Mean BMl 23.7 (sD 2.3) }\end{array}$ & \multirow{2}{*}{$\begin{array}{l}\text { Co-administered } \\
\text { with both } \\
\text { lunch and } \\
\text { dinner }\end{array}$} & $\begin{array}{l}\text { Vodka and OJ } \\
\text { Beer and low-fat foods }\end{array}$ & $\begin{array}{l}200 \\
341\end{array}$ & $\begin{array}{l}20.0 \\
19 \cdot 6\end{array}$ & $\begin{array}{l}\text { OJ and maltodextrin } \\
\text { No-alcohol beer and low- } \\
\text { fat foods }\end{array}$ & $\begin{array}{l}200 \\
341\end{array}$ \\
\hline & & & & & Beer and high-fat foods & 341 & $19 \cdot 6$ & $\begin{array}{l}\text { No-alcohol beer and } \\
\text { high-fat foods }\end{array}$ & 341 \\
\hline \multirow{4}{*}{$\begin{array}{l}\text { Westerterp- } \\
\text { Platenga } \\
\text { et al. } \\
(1999)^{(51)} \\
\text { The } \\
\text { Netherlands } \\
\text { Yeomans et al. } \\
\text { (1999) })^{(52)} \text {, UK }\end{array}$} & \multirow{2}{*}{$\begin{array}{l}\text { Five-arm, } \\
\text { randomised } \\
\text { crossover trial }\end{array}$} & \multirow{2}{*}{$\begin{array}{l}52 \text { (twenty-seven } \\
\text { females, twenty-five } \\
\text { males) }\end{array}$} & $\begin{array}{l}\text { Aged } 20-45 \\
\text { BMl between } 20.0 \text { and } 32.0\end{array}$ & \multirow{2}{*}{$\begin{array}{l}30 \text { min before } \\
\text { the food was } \\
\text { served }\end{array}$} & White wine & 340 & 29.5 & $\begin{array}{l}\text { High-fat beverage (fruit } \\
\text { juice and cream) }\end{array}$ & 340 \\
\hline & & & & & Beer & 340 & $26 \cdot 8$ & $\begin{array}{l}\text { High-protein beverage } \\
\text { (fruit juice and protein) }\end{array}$ & 340 \\
\hline & \multirow[t]{2}{*}{$\begin{array}{l}\text { Three-arm } \\
\text { crossover trial }\end{array}$} & \multirow[t]{2}{*}{24 males } & $\begin{array}{l}\text { Restrained eaters } \\
\text { Mean age } 25.4 \text { (SD 2.4) } \\
\text { Mean BMI } 23.2 \text { (SD 0.8) }\end{array}$ & \multirow[t]{2}{*}{$\begin{array}{l}20 \mathrm{~min} \text { before } \\
\text { the food was } \\
\text { served }\end{array}$} & $\begin{array}{l}\text { Carbonated alcoholic } \\
\text { apple juice }\end{array}$ & 330 & $14 \cdot 3$ & $\begin{array}{l}\text { High-carbohydrate } \\
\text { beverage (fruit juice) } \\
\text { Carbonated non- } \\
\text { alcoholic apple juice } \\
\text { Sparkling spring water }\end{array}$ & 330 \\
\hline & & & $\begin{array}{l}\text { Unrestrained eaters } \\
\text { Mean age 23.7 (SD 1.3) } \\
\text { Mean BMI } 23.2(\mathrm{SD} 0.8 \text { ) }\end{array}$ & & & & & & 330 \\
\hline \multirow[t]{2}{*}{$\begin{array}{l}\text { Yeomans et al. } \\
(2002)^{(53)}, \text { UK }\end{array}$} & \multirow[t]{2}{*}{$\begin{array}{l}\text { Three-arm, } \\
\text { crossover trial }\end{array}$} & \multirow[t]{2}{*}{18 males } & $\begin{array}{l}\text { Mean age } 24.0 \\
\text { Mean BMI } 22.8\end{array}$ & \multirow{2}{*}{$\begin{array}{l}20 \text { min before } \\
\text { the food was } \\
\text { served }\end{array}$} & \multirow[t]{2}{*}{ Lager } & \multirow[t]{2}{*}{$300 \mathrm{~g} \neq$} & \multirow[t]{2}{*}{$15 \cdot 0$} & $\begin{array}{l}\text { No-alcohol lager with } \\
\text { maltodextrin }\end{array}$ & $300 \mathrm{~g} \ddagger$ \\
\hline & & & Excluded restrained eaters & & & & & Water & $300 \mathrm{~g} \ddagger$ \\
\hline \multirow[t]{2}{*}{$\begin{array}{l}\text { Yeomans } \\
(2010)^{(54)} \text {, UK }\end{array}$} & \multirow{2}{*}{$\begin{array}{l}\text { Four-arm, } \\
\text { randomised } \\
\text { crossover trial }\end{array}$} & 40 females & $\begin{array}{l}\text { Mean age 22.0 } \\
\text { Mean BMI 22.6 }\end{array}$ & $\begin{array}{l}30 \text { min before } \\
\text { the food was }\end{array}$ & $\begin{array}{l}\text { No-alcohol beer with } \\
\text { alcohol }\end{array}$ & 300 & $15 \cdot 0$ & $\begin{array}{l}\text { No-alcohol beer with } \\
\text { maltodextrin }\end{array}$ & 300 \\
\hline & & & & served & $\begin{array}{l}\text { Carbonated fruit juice } \\
\text { with alcohol }\end{array}$ & 300 & $15 \cdot 0$ & $\begin{array}{l}\text { Carbonated fruit juice } \\
\text { with maltodextrin }\end{array}$ & 300 \\
\hline
\end{tabular}

RCT, randomised controlled trial; NR, not reported; OJ, orange juice.

* $\mathrm{ml} / \mathrm{kg}$ of body weight.

$\ddagger$ Grams of beverage was reported. 
Of the twenty-two studies, fifteen included an E-beverage comparator $^{(11,12,29,39,40,45-54)}$, nine included an NE-beverage comparator $^{(12,24,28,42,47,51-53)}$ and four included an N-beverage comparator $^{(11,41,45,51)}$. The two longer term studies ${ }^{(43,44)}$ did not specify the comparator type as participants abstained from consuming alcoholic beverages in free-living conditions. Two studies by the same author ${ }^{(28)}$ used NE-beverage comparators comprising diet soft drink with marijuana, dextroamphetamine sulphate or marijuana with the cannaboids removed.

The energy content of the E-beverage was important to take into account, and five studies ${ }^{(11,49,51,53,54)}$ included an E-beverage comparator that was energy matched with the alcoholic beverage and eleven studies ${ }^{(12,29,39,40,45-48,50,52,54)}$ included a hypoenergetic E-beverage comparator, which was lower in energy content compared with the alcoholic beverage.

\section{Participants}

A total of 706 participants were in the included studies. The minimum number of participants in a study was six ${ }^{(11)}$, while the maximum was $119^{(48)}$. There were a higher proportion of female ( $n$ 424, 60\%) than male participants ( $n$ 282, 40\%). Twelve studies ${ }^{(11,28,29,39-41,43,45,49,50,52,53)}$ recruited only male participants, and six studies recruited only female participants $^{(12,42,44,46,48,54)}$. Studies mainly recruited younger participants whose mean age ranged from $19 \cdot 6^{(42)}$ to $37 \cdot 0$ years ${ }^{(12)}$. Mean body weight was reported in five studies ${ }^{(11,24,43,44,50)}$ and it ranged from $65 \cdot 4^{(24)}$ to $78 \cdot 6 \mathrm{~kg}^{(44)}$. Where reported, the mean $\mathrm{BMI}^{(12,41,42,44,46-50,52,53,54)}$ ranged from $20 \cdot 8^{(42)}$ to $29 \cdot 8 \mathrm{~kg} / \mathrm{m}^{2(44)}$. Fourteen studies ${ }^{(11,24,29,39-45,47,48-50)}$ reported either participants' usual alcohol consumption or participants' specified usual alcohol consumption in their participant eligibility criteria, where the intake ranged from a minimum of $28.0 \mathrm{~g}$ alcohol per month ${ }^{(43,44)}$ to $267 \cdot 3 \mathrm{~g}$ alcohol per week ${ }^{(48)}$.

\section{Outcomes}

Food energy intake and the total energy intake for the included studies are presented in the online Supplementary Table S2.

\section{Food energy intake}

Participants' food energy intake was reported by twelve studies $^{(11,12,24,28,41,42,45,47,51-53)}$ when compared with NE- or N-beverage comparator conditions. Alcohol consumption increased food energy intake in three studies ${ }^{(41,42,45)}$ and remained unchanged in eight ${ }^{(12,24,28,47,51-53)}$. One study ${ }^{(11)}$ demonstrated a dose-dependant effect, with no change in food energy intake with a low-dose alcoholic beverage, but a significant decrease with the high-dose alcoholic beverage.

Of the fifteen studies ${ }^{(11,12,29,39,40,45-54)}$ that compared an alcohol dose against an E-beverage on food energy intake, seven $^{(40,45,46,49,51,53,54)}$ reported a significant increase in food energy intake and eight studies ${ }^{(11,12,29,39,47,48,50,52)}$ reported no significant change. Of the five studies ${ }^{(11,49,51,53,54)}$ utilising an isoenergetic non-alcoholic comparator, four ${ }^{(49,51,53,54)}$ showed that alcohol consumption increased food energy intake, when compared with the comparator. Of the thirteen studies $^{(11,29,39,40,45,46,48-54)}$ that compared a low-dose alcoholic beverage $(<30 \mathrm{~g}$ or $<0.6 \mathrm{~g} / \mathrm{kg})$ with E-beverages, seven studies ${ }^{(40,45,46,49,51,53,54)}$ demonstrated that food energy intake increased with consumption of a low-dose alcoholic beverage when compared with E-beverages and six studies ${ }^{(11,29,39,48,50,52)}$ showed no significant change. In contrast, of the four studies ${ }^{11,12,39,47)}$ that compared a high-dose alcoholic beverage ( $\geq 30 \mathrm{~g}$ or $\geq 0.6 \mathrm{~g} / \mathrm{kg}$ ) with E-beverages, all four studies ${ }^{(11,12,39,47)}$ demonstrated no significant change in food energy intake.

Twelve studies ${ }^{(12,24,39-42,45,46,49,52-54)}$ involving 417 participants reported mean data on food energy intake for both intervention and comparator groups enabling them to be included in a meta-analysis using a random-effects model. Alcoholic beverages resulted in statistically significant higher food energy intake compared with non-alcoholic beverage comparators (weighted mean difference $343 \mathrm{~kJ}, 95 \%$ CI 161 , $525 \mathrm{~kJ}$ ) (Fig. 2). Statistically significant heterogeneity and a substantial amount of inconsistency were observed. Sensitivity analysis using the HKSJ method ${ }^{(27)}$ resulted in wider CI, although this did not change our conclusion (weighted mean difference $343 \mathrm{~kJ}, 95 \%$ CI 109, $577 \mathrm{~kJ}$ ).

The asymmetrical funnel plots and Egger's regression test suggests small-study effects may exist in the meta-analysis for food energy intake (Egger's test: $P=0.002$ ) (online Supplementary Fig. S1). There are several possible reasons for this asymmetry, including reporting biases, or clinical and methodological heterogeneity. A sensitivity analysis conducted using correlation coefficients of 0.2 and 0.8 to estimate the standard error of the mean difference in crossover trials did not substantially change the results of the meta-analysis. The influence analysis demonstrated that removing each study one by one did not substantially alter the overall effect (online Supplementary Table S3).

\section{Total energy intake}

Total energy intake (the sum of both the beverage and food consumption) was reported in eight studies $(11,12,24,41,42,47,51,53)$ that compared the alcohol dose with either an NE-beverage or an N-beverage. Seven of those studies ${ }^{(11,12,24,41,42,51,53)}$ showed a significant increase in the total energy intake with alcohol consumption, and one study ${ }^{(47)}$ demonstrated no change with low-dose (31.3 g alcohol) but a significant increase with highdose ( $54 \mathrm{~g}$ alcohol) alcoholic beverages. Of the seven studies that increased the total energy intake, five ${ }^{(11,12,24,41,42)}$ utilised an alcohol dose $\geq 30 \mathrm{~g}$ or $\geq 0 \cdot 6 \mathrm{~g} / \mathrm{kg}$.

Total energy intake was reported in nine studies $^{(11,12,29,39,40,47,50,51,53)}$ that compared the alcohol dose with an E-beverage. Alcohol consumption increased the total energy intake compared with E-beverages in two studies ${ }^{(40,53)}$, no significant change was observed in three studies ${ }^{11,29,51)}$, and both a significant increase in total energy intake and no significant change was observed in four studies ${ }^{(12,39,47,50)}$.

Two separate studies conducted by Cordain et al. ${ }^{(43,44)} \mathrm{did}$ not specify the type of non-alcoholic beverage comparator as these were both long-term free-living studies, however, they reported no significant change in the total energy intake between the alcohol consumption and abstinence periods. 


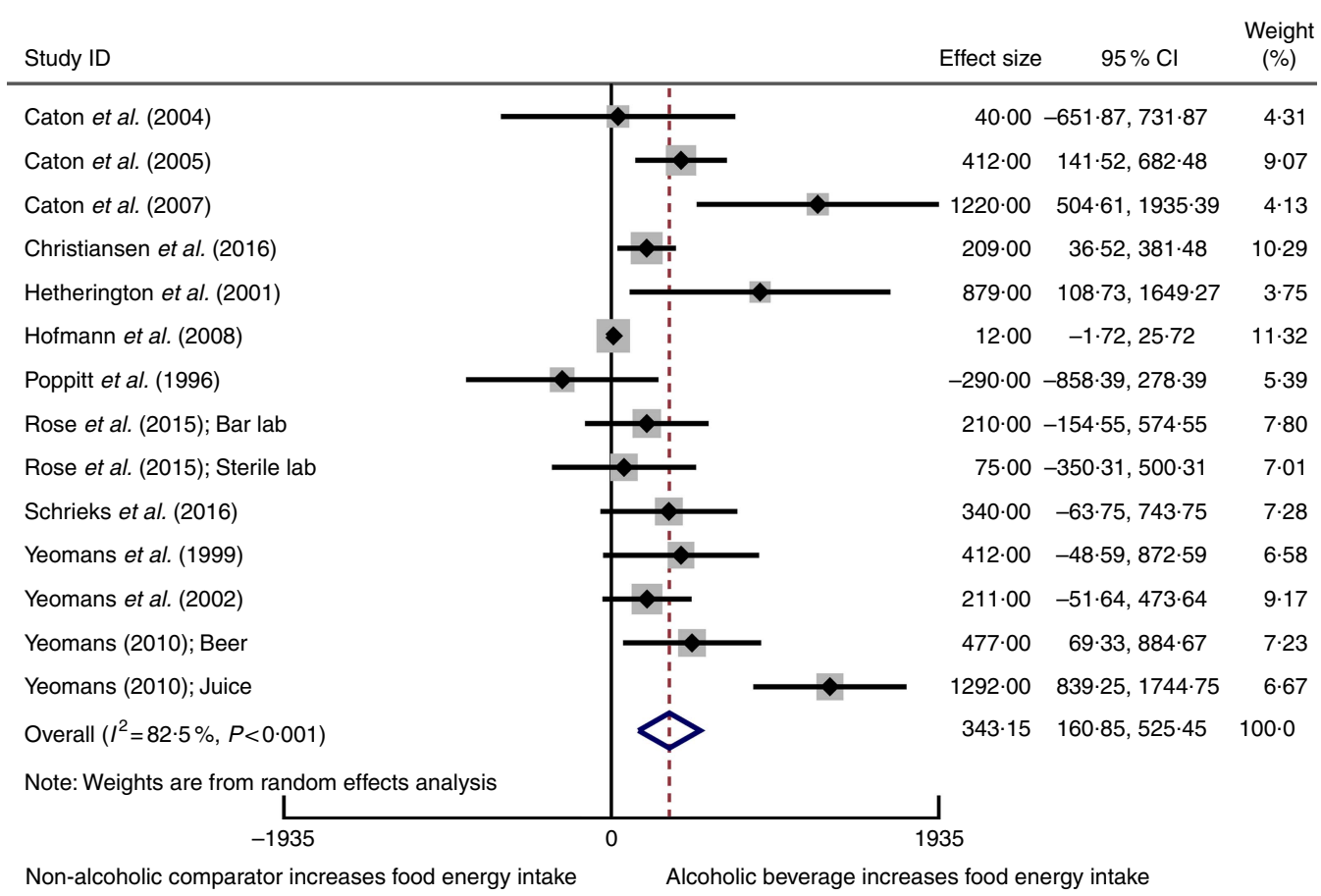

Fig. 2. Forest plot for changes in food energy intake $(\mathrm{kJ})$ as a result of alcoholic beverage consumption using the random-effects model. The black squares represent the mean difference from each study, the grey squares represent the weight assigned to that study, while the left and right extremes of the squares represent the corresponding $95 \% \mathrm{Cl}$. The hollow diamond represents the overall pooled effects while the left and right points of the diamond represent the corresponding $95 \% \mathrm{Cl}$.

None of the studies reported a decrease in food energy intake or total energy intake following consumption of alcohol.

Eight studies ${ }^{(12,24,39-42,52,53)}$ involving 269 participants reported data on total energy intake and were pooled and included in the meta-analysis. Total energy intake was increased with consumption of an alcoholic beverage compared with a non-alcoholic beverage or no beverage (weighted mean difference $1072 \mathrm{~kJ}, 95 \%$ CI 820, 1323kJ) (Fig. 3). When compared with a non-alcoholic beverage, consumption of an alcoholic beverage resulted in an increase in total energy intake. Statistically significant heterogeneity and a substantial amount of inconsistency were observed. The $95 \%$ CI for the meta-analysis when using the HKSJ method ${ }^{(27)}$ were wider, although this did not alter the conclusion (weighted mean difference $1072 \mathrm{~kJ}, 95 \%$ CI 693, $1450 \mathrm{~kJ}$ ).

No evidence of small-study effects in the meta-analysis of total energy intake was observed, based on visual inspection of a funnel plot and Egger's regression test $(P=0 \cdot 8)$ (online Supplementary Fig. S2). A sensitivity analysis conducted using correlation coefficients of 0.2 and 0.8 to estimate the standard error of the mean difference in crossover trials did not substantially change the results in the meta-analysis. The influence analysis demonstrated that removing each study one by one did not substantially alter the overall effect (online Supplementary Table S4).

Impact of comparator type, alcohol dose and sex on food energy intake and total energy intake

To explore the effects of comparator drink (NE-, N- and E-beverages), alcohol dose (defined by low and high alcohol dose) and sex (male only studies and female only studies), subgroup analyses were performed (Table 4). The subgroup analyses for comparator type indicated that alcohol consumption significantly increased food energy intake when compared with NE- and N-beverages as well as E-beverages. While food energy intake increased to a greater extent with alcohol consumption when E-beverages were compared with NE- and N-beverages, overlapping 95\% CI were observed. Alcohol consumption significantly increased the total energy intake when compared with both NE- and $\mathrm{N}$-beverages and E-beverages. The total energy intake increased to a greater extent with alcohol consumption when NE- and N-beverages were compared with E-beverages; however, overlapping $95 \%$ CI were observed. For food energy intake, both low-dose and high-dose alcohol increased food energy intake. Low-dose alcohol increased food energy intake to a greater extent compared with highdose alcohol, although overlapping 95\% CI were observed. Both low-dose and high-dose alcohol increased the total energy intake, when compared with non-alcoholic comparators. High-dose alcohol increased to a greater extent than low-dose alcohol, although overlapping 95\% CI were observed. Subgroup analyses for sex demonstrated that with both male-only studies and female-only studies, alcohol consumption significantly increased food energy intake, when compared with non-alcoholic comparators. Food energy intake was increased to a greater extent with alcohol consumption in male-only studies when compared with female-only studies; however, overlapping 95\% CI were observed. Both male-only studies and female-only studies observed a significant increase in the total energy intake 


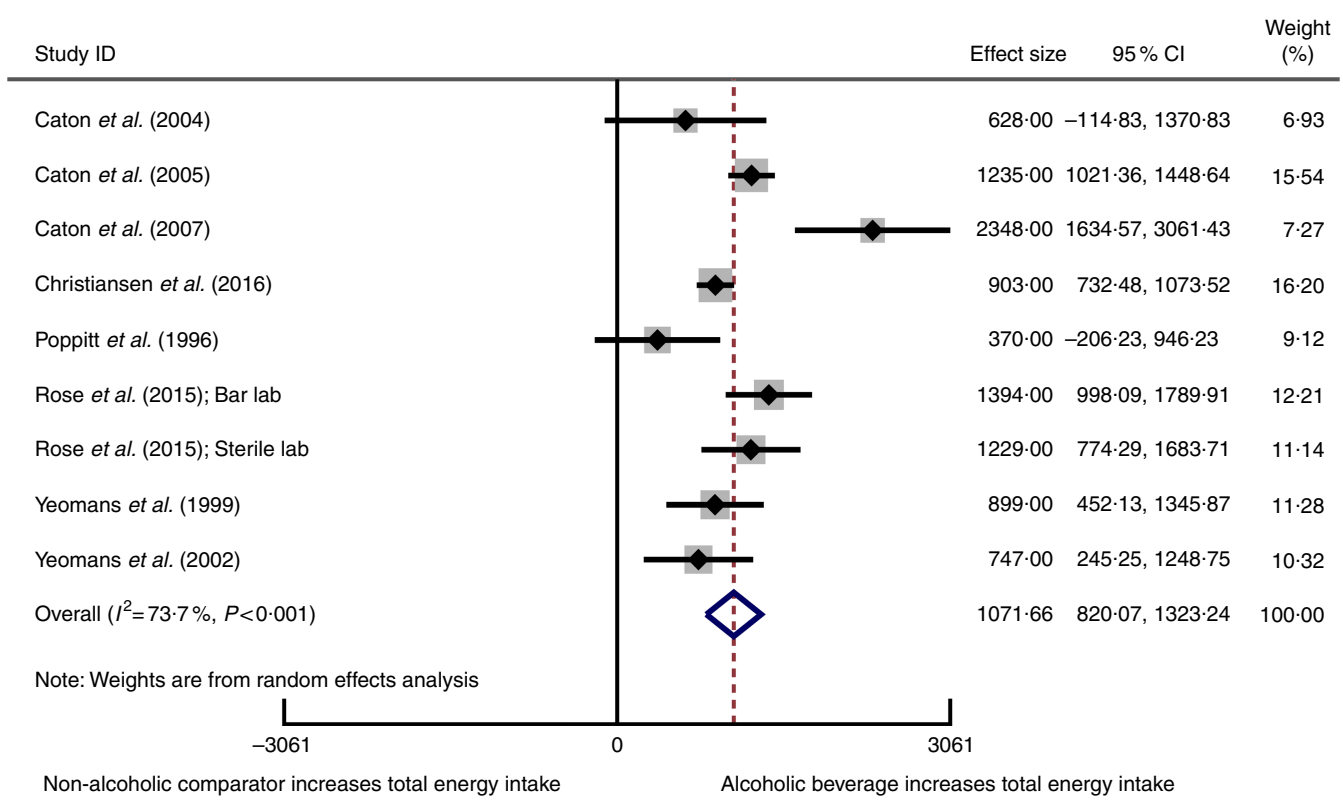

Fig. 3. Forest plot for changes in total energy intake $(\mathrm{kJ})$ as a result of alcoholic beverage consumption using the random-effects model. The black squares represent the mean difference from each study, the grey squares represent the weight assigned to that study, while the left and right extremes of the squares represent the corresponding $95 \% \mathrm{Cl}$. The hollow diamond represents the overall pooled effects while the left and right points of the diamond represent the corresponding $95 \% \mathrm{Cl}$.

when compared with non-alcoholic comparators. Similar to food energy intake, total energy intake was increased to a greater extent with alcohol consumption in male-only studies when compared with female-only studies; however, overlapping $95 \%$ CI were observed.

\section{Body weight change}

Body weight change was reported in three studies which had a duration of between $13 \mathrm{~d}$ and 10 weeks $^{(11,43,44)}$. Two of the studies reported no significant differences in body weight change between the alcoholic intervention period, abstention period and baseline ${ }^{(43,44)}$. One study reported that weight increased significantly by a mean of 0.9 (SE 0.4 ) $\mathrm{kg}$ between the first and last day, although this weight change could not be directly attributable to the consumption of a specific beverage $^{(11)}$

\section{Quality assessment and risk of bias}

Eighteen studies ${ }^{(11,12,24,29,39-45,47,49-54)}$ achieved a strong rating as defined by the EPHPP quality assessment tool, four studies ${ }^{(28,46,48)}$ were moderate and no studies were identified as weak (Fig. 4 and online Supplementary Table S5). Inadequate reporting of the randomisation method was the main reason for lower quality ratings. Nine studies ${ }^{(24,39,40,42,47,48,52-54)}$ did not report a source of funding that would present a conflict of interest, and five studies ${ }^{(12,29,43,44,51)}$ reported either industry funding or industry supply of alcohol for the study. Only two experiments $^{(28)}$ used double-blind conditions, while twelve studies $^{(11,12,29,39,40,42,45-50)}$ were single-blinded. Single-blind conditions were achieved by disguising either the true nature of the study to participants or the non-alcoholic beverage or a combination of both.

\section{Discussion}

To our knowledge, this is the first systematic review with metaanalyses performed for all available RCT and non-randomised crossover studies that compared the effect of alcohol consumption on both food energy intake and total energy intake in humans. All twenty-two studies consistently demonstrated that participants did not reduce their food energy intake to compensate for the energy consumed from alcoholic beverages.

\section{Food energy intake}

Previously, it has been suggested that alcoholic beverage consumption may increase food energy ${ }^{(10)}$. In this review, the studies indicate that energy consumption as food is acutely increased by an average of $343 \mathrm{~kJ}$ after alcohol consumption when compared with non-alcoholic beverages. These results could be attributed to a combination of mechanisms. Alcohol's effects on human expectancy (previously learned associations between alcohol consumption and appetite stimulation), disinhibiting eating restraint and the satiety hormones have been proposed as possible mechanisms for increased food intake with alcohol consumption ${ }^{(3,10,55)}$. However, others have suggested that these mechanisms are unlikely to induce the proposed stimulation of alcohol consumption on appetite and potential increased food intake in human participants ${ }^{(10)}$. Rather, alcohol's pharmacological effects on several neurotransmitters in the central nervous system that influence 
Table 4. Meta-analyses of food energy intake and total energy intake with sub-groups defined by 'low alcohol' and 'high alcohol' dose

\begin{tabular}{|c|c|c|c|c|c|c|c|c|c|}
\hline Outcomes & Subgroup & $\begin{array}{l}\text { No. of } \\
\text { trials }\end{array}$ & Participants & Statistical method & $\begin{array}{c}\text { Test of } \\
\text { heterogeneity }(P)\end{array}$ & $\begin{array}{l}\text { Heterogeneity } \\
\left(P^{\prime}\right), \%\end{array}$ & $\begin{array}{l}\text { Net change } \\
(\mathrm{kJ})\end{array}$ & $95 \% \mathrm{Cl}$ & $\begin{array}{l}P \text { for meta- } \\
\text { analysis }\end{array}$ \\
\hline Food energy intake & $\begin{array}{l}\mathrm{N} \text { - and NE-beverage } \\
\text { comparators }\end{array}$ & 8 & 271 & $\begin{array}{l}\text { Mean difference (IV, random, } \\
95 \% \mathrm{Cl} \text { ) }\end{array}$ & 0.04 & 52 & 215.03 & $16 \cdot 53,413.53$ & 0.03 \\
\hline Food energy intake & E-beverage comparators & 10 & 232 & $\begin{array}{l}\text { Mean difference (IV, random, } \\
95 \% \mathrm{Cl} \text { ) }\end{array}$ & $<0.001$ & 87 & 410.46 & $143.91,677.02$ & 0.003 \\
\hline Total energy intake & $\begin{array}{l}\mathrm{N} \text { - and NE- beverage } \\
\text { comparators }\end{array}$ & 7 & 245 & $\begin{array}{l}\text { Mean difference (IV, random, } \\
95 \% \mathrm{Cl} \text { ) }\end{array}$ & 0.002 & 71 & $1150 \cdot 46$ & $857 \cdot 81,1443 \cdot 11$ & $<0.001$ \\
\hline Total energy intake & E-beverage comparators & 5 & 84 & $\begin{array}{l}\text { Mean difference (IV, random, } \\
95 \% \mathrm{Cl})\end{array}$ & 0.003 & 74 & $746 \cdot 75$ & $338.82,1154 \cdot 69$ & $<0.001$ \\
\hline Food energy intake & $\begin{array}{l}\text { Low alcohol dose }(<30 \mathrm{~g} \text { or } \\
\quad<0.6 \mathrm{~g} / \mathrm{kg})\end{array}$ & 9 & 212 & $\begin{array}{l}\text { Mean difference (IV, random, } \\
95 \% \mathrm{Cl} \text { ) }\end{array}$ & $<0.001$ & 86 & 388.94 & $121 \cdot 85,656 \cdot 03$ & 0.004 \\
\hline Food energy intake & $\begin{array}{l}\text { High alcohol dose ( } \geq 30 \mathrm{~g} \text { or } \\
\geq 0.6 \mathrm{~g} / \mathrm{kg} \text { ) }\end{array}$ & 6 & 217 & $\begin{array}{l}\text { Mean difference (IV, random, } \\
95 \% \mathrm{Cl} \text { ) }\end{array}$ & 0.04 & 57 & $246 \cdot 26$ & $-17 \cdot 17,509.69$ & 0.07 \\
\hline Total energy intake & $\begin{array}{l}\text { Low alcohol dose }(<30 \mathrm{~g} \text { or } \\
<0.6 \mathrm{~g} / \mathrm{kg})\end{array}$ & 4 & 64 & $\begin{array}{l}\text { Mean difference (IV, random, } \\
95 \% \mathrm{Cl} \text { ) }\end{array}$ & 0.001 & 81 & $759 \cdot 29$ & $263.79,1254.79$ & 0.003 \\
\hline Total energy intake & $\begin{array}{l}\text { High alcohol dose ( } \geq 30 \mathrm{~g} \text { or } \\
\geq 0.6 \mathrm{~g} / \mathrm{kg} \text { ) }\end{array}$ & 6 & 217 & $\begin{array}{l}\text { Mean difference (IV, random, } \\
95 \% \mathrm{Cl} \text { ) }\end{array}$ & $<0.001$ & 80 & $1229 \cdot 37$ & $830 \cdot 25,1628 \cdot 49$ & $<0.001$ \\
\hline Food energy intake & Males only & 7 & 125 & $\begin{array}{l}\text { Mean difference (IV, random, } \\
95 \% \mathrm{Cl} \text { ) }\end{array}$ & 0.15 & 37 & $407 \cdot 92$ & $200 \cdot 39,615 \cdot 46$ & $<0.001$ \\
\hline Food energy intake & Females only & 5 & 178 & $\begin{array}{l}\text { Mean difference (IV, random, } \\
95 \% \mathrm{Cl} \text { ) }\end{array}$ & $<0.001$ & $90 \cdot 4$ & $321 \cdot 17$ & $-0.48,642 \cdot 82$ & 0.05 \\
\hline Total energy intake & Males only & 5 & 75 & $\begin{array}{l}\text { Mean difference (IV, random, } \\
95 \% \mathrm{Cl} \text { ) }\end{array}$ & 0.002 & $76 \cdot 5$ & $1146 \cdot 56$ & $712 \cdot 30,1580 \cdot 83$ & $<0.001$ \\
\hline Total energy intake & Females only & 2 & 80 & $\begin{array}{l}\text { Mean difference (IV, random, } \\
95 \% \mathrm{Cl} \text { ) }\end{array}$ & 0.082 & $66 \cdot 9$ & $710 \cdot 48$ & $208 \cdot 69,1212 \cdot 28$ & 0.006 \\
\hline
\end{tabular}

$\mathrm{N}$-beverage, no beverage; NE-beverage, beverages that contained no or negligible energy; E-beverage, energy-containing beverages. 
Selection bias

Study design

Confounders

Blinding

Data collection methods

Withdrawals and drop-outs

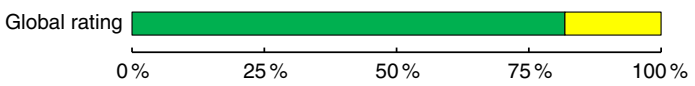

Fig. 4. Summary of the Effective Public Heath Practice Project quality assessment tool for quantitative studies within the included studies across the seven domains: selection bias, study design, confounders, blinding, data collection methods, withdrawals and drop-outs and global rating. The proportion of included studies with each judgement: $\square$, strong rating; $\square$, moderate rating; $\square$, weak rating.

behaviour may explain this effect ${ }^{(10,56)}$. Alcohol binds to $\mathrm{GABA}_{\mathrm{A}}$ receptors at low to moderate doses, which is involved in the control of food intake in mice ${ }^{(9,56,57)}$. In vitro and animal studies have also shown that alcohol consumption stimulates the release of opioid peptides, which has been postulated to be associated with orosensory reward aspects that influence food intake ${ }^{(58,59)}$.

The only study ${ }^{39)}$ in this review that investigated a doseresponse relationship between alcohol and food consumption reported that alcohol increased food energy intake only after consumption of $32 \mathrm{~g}$ of alcohol. Conversely, the present review found no increase in food energy intake after consumption of a high alcohol dose ( $\geq 30 \mathrm{~g}$ or $\geq 0.6 \mathrm{~g} / \mathrm{kg}$ ) when compared with NE- or N-beverages and E-beverages. The discrepancy in findings may be due to the differences in methodologies among the reviewed studies as well as the review pooling available data from the included studies. Reviewed studies used both fixed and adjusted alcohol, different alcohol doses and types of alcohol. However, the highest fixed dose of alcohol used in a study was $54 \mathrm{~g}^{(47)}$ and the highest adjusted alcohol dose was $0.75 \mathrm{~g} / \mathrm{kg}^{(28)}$. In $2016,26.9 \%$ of the UK drinking population reported binge drinking alcohol on their heaviest drinking day, which means that males and females had consumed in excess of 64 and $48 \mathrm{~g}$ of alcohol, respectively ${ }^{(60)}$. This is greater than the included review's highest alcohol doses of $54 \mathrm{~g}$ and $0.75 \mathrm{~g} /$ $\mathrm{kg}$ in one sitting, for fixed dose and adjusted dose, respectively. As this review's findings are inconsistent with the findings with one study ${ }^{(39)}$ that investigated a dose-response relationship, further research is warranted to investigate a possible dose-response relationship between alcohol consumption and food energy intake. Differences in study populations, types of alcoholic beverages provided and the usual eating habits could vastly influence the dose-response relationship observed ${ }^{(39)}$.

In addition, the combination of the type of alcohol ingested and the types of food served may influence results. It has previously been suggested that consumers value food pairings with wine, although this was less important with beer ${ }^{(61)}$, which was reported to pair well with 'junk or snack-type food'. Food that were identified to be consumed with beer were snacks or convenience foods such as nuts and crisps ${ }^{(61)}$. In the studies reviewed here, beer was predominantly used as the intervention alcoholic beverage, although the food provided varied. More research needs to be performed to see whether types of alcoholic beverages and their consumption with specific types of food influence food energy intake. For example, if drinkers were provided a choice when they drink beer, it would be insightful to investigate whether they prefer to consume more or less energy-dense foods.

\section{Total energy intake}

The present review demonstrated that alcohol consumption, when compared with non-alcoholic NE- or N-beverages, increased the total energy intake by an average of $1072 \mathrm{~kJ}$, which is the sum of food energy and beverage energy intake. However, due to inconsistent reporting of total energy intake within the five studies that used isoenergetic non-alcoholic E-beverages comparators, no associations were able to be determined. All twenty-two reviewed studies consistently reported that participants did not compensate for the energy that was consumed as part of the alcoholic beverage by eating less food, which was similarly reported by Yeomans ${ }^{(10)}$.

An increase in total energy intake following consumption of alcohol is supported in a previous meta-analysis ${ }^{(16)}$. The high energy content of the alcoholic beverages used in the intervention conditions contributes to the increase in total energy intake reported in the studies and therefore is more evident when compared with the negligible energy content of NEbeverages or N-beverages.

The subgroup analyses showed that both low-dose $(<30 \mathrm{~g}$ or $<0.6 \mathrm{~g} / \mathrm{kg}$ ) and high-dose ( $\geq 30 \mathrm{~g}$ or $\geq 0.6 \mathrm{~g} / \mathrm{kg}$ ) alcoholic beverages increased the total energy intake, although high-dose alcoholic beverages increased it to a greater extent; however, overlapping 95\% CI were observed. This present review suggests that high alcohol dose may stimulate total energy intake, when compared with $\mathrm{N}$ - or NE-beverage, as five of the eight studies that increased the total energy intake used a high alcohol dose. The observed increase in total energy intake is likely due to the higher energy content of the high-dose alcoholic beverages compared with the low-dose alcoholic beverages. Interestingly, when considering epidemiological studies and the relationship between alcohol consumption levels and body weight, only heavy alcohol consumption is associated with increased body weight in males ${ }^{(4)}$. It is likely this was due to the limited reporting of total energy intake in low alcohol dose studies and the inclusion of the two studies that used a low alcohol dose with relatively longer study durations. Furthermore, the finding that total energy intake increased in response to high alcohol dose to a greater extent than low alcohol dose was not supported when the high alcohol doses were compared with E-beverages. This suggests that E-beverages also increase the total energy intake, which may negate any marked differences in the increased total energy intake when compared with alcoholic beverages. The review and subgroup meta-analyses suggest that alcohol consumption increases the total energy intake, with the review's 
findings suggesting that high-dose alcohol has a stronger effect on increasing the total energy intake than the low-dose alcohol.

\section{Implications}

As obesity continues to increase globally, there is a need to understand the impact of habitually consuming energy-dense alcohol. Passive overconsumption of dietary energy, with poor compensation for energy intake consumed as alcoholic beverages, may lead to positive energy balance and promote weight gain in people who regularly consume alcohol. Previous reviews have reported conflicting evidence on the relationship between the consumption of alcoholic beverages in general ${ }^{(4)}$ or specifically beer ${ }^{(5)}$ and body weight. Alcohol dose and type of beverage may also be a confounding factor that is evident in two crossover studies included in the present review. These studies measured changes in body weight with alcohol consumption and failed to detect any changes after 6 weeks ${ }^{(43)}$ of daily and 10 weeks $^{(44)}$ of $5 \mathrm{~d}$ /week of red wine consumption $(27.6 \mathrm{~g}$ alcohol $/ \mathrm{d})$. Over longer periods of time, wine consumption has been reported to be protective against weight gain, which could be attributed to its resveratrol content, whereas consumption of beer and spirits has been positively associated with weight gain, particularly at heavy consumption levels ${ }^{(4)}$.

Our findings suggest alcohol consumption can likely predispose individuals to energy imbalance by increasing both acute food energy intake and total energy intake. This was not only evident for consumption of low alcohol doses but for higher doses as well. Although further investigation with additional high-quality studies to confirm the effect of alcohol consumption on food energy intake is strongly warranted, the present review has implications for alcohol consumption guidelines. Alcohol consumers need to be aware that whether they fail to compensate for the energy ingested from alcohol the next day, this becomes an additional source of energy that will influence the overall energy balance. These public health messages have to focus on the settings where alcoholic beverages are commonly consumed, such as restaurants, pubs, sporting events and at home. Furthermore, these messages can be directed at vulnerable population groups who frequently consume alcoholic beverages or frequently consume a high number of alcoholic beverages on single occasions and who may also be at risk of weight-related problems due to the low socioeconomic status. The vulnerable population groups in both Great Britain and Australia are young adults and middle-aged adults who are particularly at $\operatorname{risk}^{(60,62,63)}$.

\section{Strengths}

This is the first systematic review and meta-analyses to specifically investigate alcohol consumption and quantify its effect on both food energy intake and total energy intake. Food energy intake has not previously been investigated in a systematic review or meta-analysis. A broad scoping search was applied to four databases, which retrieved an extensive number of records and with no date limits applied with the search strategy, the authors are confident that the search strategy retrieved all relevant studies.

\section{Limitations}

A number of limitations were identified. First, studies were conducted predominantly with young adult female participants and hence, the generalisability of these studies to other age groups, such as older males and females, is limited. Second, reporting of participant baseline characteristics, such as age, BMI, usual alcohol consumption levels and ethnicity, was not consistent across the twenty-two studies. Third, the cut-offs used to classify unrestrained and restrained eaters were markedly different for studies that measured this factor, despite using similar questionnaires such as the DEBQ, TFEQ or their respective restraint subscales. Finally, the definitions regarding low, moderate and high usual alcohol consumption levels were not consistent across studies as they were conducted in different countries with different interpretations for the alcohol content of a standard drink and different guidelines for consumption.

Experimental designs and methodologies differed considerably, which created challenges in comparing studies and the synthesis of data. A range of different alcoholic beverage types and doses were utilised for the interventions. Blood alcohol concentration (BAC) is influenced by the alcohol type, dose and rate of drinking. As an example, consumption of spirits are shown to result in a higher BAC than wine and beer, despite ingestion of the same alcohol dose $^{(64)}$. Furthermore, associations have been demonstrated between spirits and beer and body weight, which are stronger than the associations found between wine and body weight $^{(4)}$. The impact of alcohol on body weight is further influenced by characteristics such as sex, alcohol type and total consumption patterns ${ }^{(4,5)}$. Despite this, many factors, known to have an impact on body weight, have not been accounted for in previous studies ${ }^{(3)}$; such as sex, physical activity levels and sleep habits and, therefore, may contribute to the lack of a clear demonstrable relationship between alcohol consumption and body weight. In addition, the use of deception and disguising of the non-alcoholic beverages was utilised irregularly across studies.

The test food provided varied between studies, with different savoury and sweet foods used, such as chocolate snacks and pasta meals. Also, the primary outcomes of food energy intake and the total energy intake were not consistently reported. Lastly, very few studies measured the participants' liking of the food provided, which may have influenced the amount of food they consumed ${ }^{(65,66)}$.

At the review level, limitations are that non-English-language studies were excluded from the search strategy, which may have resulted in language bias. Furthermore, a number of studies that investigated the effects of alcohol consumption on the intake of liquid meals were also excluded. No hand searching of the reference list of the included studies was conducted due to the extensive number of records retrieved. There are several potential limitations with the meta-analysis. 
First, since this was an aggregate data meta-analysis, there is potential for ecological fallacy, specifically Simpson's Paradox, to exist ${ }^{(67)}$. Second, some of the statistically significant findings may have been due to chance, given the large number of statistical tests that were conducted. Third, as the studies were not randomly assigned to covariates or subgroups in the metaanalysis, they are considered to be observational in nature, and the results of the subgroup analyses do not support causal inferences. Lastly, some of the sub-group meta-analyses were conducted on the basis of small numbers of studies with high $I^{2}$ values, which may have influenced the findings.

\section{Further work/directions}

The reviewed studies predominantly utilised a fixed alcohol dose although very few studies included an isoenergetic nonalcoholic comparator. To determine whether the effect on food energy intake is attributable to the pharmacological effects of alcohol rather than the energy content of the alcoholic beverage, the use of an isoenergetic non-alcoholic comparator is recommended. Furthermore, for research studies that utilise a broad inclusion criteria for age and BMI, it is recommended to utilise adjusted alcohol dose $(\mathrm{g} / \mathrm{kg}$ body weight), rather than fixed alcohol dose, to reflect differences in body size and alcohol metabolism. Standardisation of study methods, such as the type of alcohol used for the beverages and foods used and to test in a range of different age groups, is also warranted. The consumption of beer and spirits is positively associated with weight gain to a greater extent compared with wine intake ${ }^{(4)}$. Consistent reporting of both food energy intake and total energy intake outcomes and participant characteristics is fundamental to provide additional evidence for meta-analyses. Lastly, consideration of the participants' liking of the test food with food liking questionnaires is crucial to minimise a potential confounding factor for energy intake.

\section{Conclusion}

This review demonstrates that compared with non-alcoholic beverages or no beverage, the consumption of alcoholic beverages significantly increases both food energy intake and total energy intake by 343 and $1072 \mathrm{~kJ}$ on average, respectively. This quantity of energy has implications for the body weight of those who consume alcoholic beverages and food provision in the settings in which they do so, as chronic passive overconsumption of alcoholic beverages will add fuel to the obesity epidemic.

\section{Acknowledgements}

The authors would like to acknowledge Dr StellaMay Gwini (biostatistician) for her assistance with the meta-analysis component of this publication, Ms Cassandra Freeman (subject librarian) for her assistance with the search strategy and Ms Claire Bristow for her assistance with retrieving papers.

A. K. is supported by an Australian Government Research Training Program Scholarship. G. P. was supported by a
Monash University Vacation Scholarship Program. M. J. P. is supported by an Australian National Health and Medical Research Council Early Career Fellowship.

A. K. and H. T. conceptualised the analytical protocol. A. K. conducted the literature search. A. K. and G. P. assisted with table and figure creation and extracted data. A. K., A. L. D., G. P. and M. J. P. conducted the study data analyses. A. K. and M. J. P. conducted the meta-analyses. A. K. was the primary writer. H. T., A. L. D., G. P. and M. J. P. reviewed the content of the manuscript and provided editorial feedback.

None of the authors have any conflicts of interest to declare.

\section{Supplementary material}

For supplementary material/s referred to in this article, please visit https://doi.org/10.1017/S0007114518003677

\section{References}

1. World Health Organization (2014) Global Status Report on Noncommunicable Diseases 2014. Geneva: World Health Organization.

2. World Health Organization (2014) Global Status Report on Alcohol and Health 2014. Geneva: World Health Organization.

3. Traversy G \& Chaput J-P (2015) Alcohol consumption and obesity: an update. Curr Obes Rep 4, 122-130.

4. Sayon-Orea C, Martinez-Gonzalez MA \& Bes-Rastrollo M (2011) Alcohol consumption and body weight: a systematic review. Nutr Rev 69, 419-431.

5. Bendsen NT, Christensen R, Bartels EM, et al. (2013) Is beer consumption related to measures of abdominal and general obesity? A systematic review and meta-analysis. Nutr Rev $\mathbf{7 1}$, 67-87.

6. Albani V, Bradley J, Wrieden WL, et al. (2018) Examining associations between body mass index in 18-25 year-olds and energy intake from alcohol: findings from the Health Survey for England and the Scottish Health Survey. Nutrients 10, 1147.

7. Bagnardi V, Rota M, Botteri E, et al. (2014) Alcohol consumption and site-specific cancer risk: a comprehensive doseresponse meta-analysis. Br J Cancer 112, 580-593.

8. Ronksley PE, Brien SE, Turner BJ, et al. (2011) Association of alcohol consumption with selected cardiovascular disease outcomes: a systematic review and meta-analysis. BMJ 342, d671.

9. Yeomans MR, Caton S \& Hetherington MM (2003) Alcohol and food intake. Curr Opin Clin Nutr Metab Care 6, 639-644.

10. Yeomans MR (2010) Alcohol, appetite and energy balance: is alcohol intake a risk factor for obesity? Physiol Behav 100, 82-89.

11. Foltin RW, Kelly TH \& Fischman MW (1993) Ethanol as an energy source in humans: comparison with dextrosecontaining beverages. Appetite 20, 95-110.

12. Poppitt SD, Eckhardt JW, McGonagle J, et al. (1996) Shortterm effects of alcohol consumption on appetite and energy intake. Physiol Behav 60, 1063-1070.

13. Almiron-Roig E, Palla L, Guest K, et al. (2013) Factors that determine energy compensation: a systematic review of preload studies. Nutr Rev 71, 458-473.

14. Blundell J, de Graaf C, Hulshof T, et al. (2010) Appetite control: methodological aspects of the evaluation of foods. Obes Rev 11, 251-270.

15. Yeomans MR (2004) Effects of alcohol on food and energy intake in human subjects: evidence for passive and active over-consumption of energy. Br J Nutr 92, Suppl. 1, S31-S34. 
16. Chapman CD, Benedict C, Brooks SJ, et al. (2012) Lifestyle determinants of the drive to eat: a meta-analysis. Am J Clin Nutr 96, 492-497.

17. Gee C (2006) Does alcohol stimulate appetite and energy intake? Br J Community Nurs 11, 298-302.

18. Griffiths P (2002) Evidence informing practice: introducing the mini-review. Br J Community Nurs 7, 38-39.

19. Moher D, Liberati A, Tetzlaff J, et al. (2009) Preferred reporting items for systematic reviews and meta-analyses: the PRISMA statement. BMJ 339, b2535.

20. National Health and Medical Research Council (2009) NHMRC Additional Levels of Evidence and Grades for Recommendations for Developers of Guidelines. Melbourne: NHMRC.

21. Thomas BH, Ciliska D, Dobbins M, et al. (2004) A process for systematically reviewing the literature: providing the research evidence for public health nursing interventions. Worldviews Evid Based Nurs 1, 176-184.

22. Kalinowski A \& Humphreys K (2016) Governmental standard drink definitions and low-risk alcohol consumption guidelines in 37 countries. Addiction 111, 1293-1298.

23. National Health and Medical Research Council (2009) Australian Guidelines to Reduce Health Risks from Drinking Alcohol. Canberra: NHMRC.

24. Rose A, Hardman C \& Christiansen P (2015) The effects of a priming dose of alcohol and drinking environment on snack food intake. Appetite 95, 341-348.

25. Rose AK \& Duka T (2006) Effects of dose and time on the ability of alcohol to prime social drinkers. Behav Pharmacol 17, 61-70.

26. DerSimonian R \& Laird N (1986) Meta-analysis in clinical trials. Control Clin Trials 7, 177-188.

27. IntHout J, Ioannidis JP \& Borm GF (2014) The HartungKnapp-Sidik-Jonkman method for random effects metaanalysis is straightforward and considerably outperforms the standard DerSimonian-Laird method. BMC Med Res Methodol 14, 25.

28. Hollister LE (1971) Hunger and appetite after single doses of marihuana, alcohol, and dextroamphetamine. Clin Pharmacol Ther 12, 44-49.

29. Buemann B, Toubro S \& Astrup A (2002) The effect of wine or beer versus a carbonated soft drink, served at a meal, on ad libitum energy intake. Int J Obes Relat Metab Disord 26, 1367-1372.

30. Tremblay A \& St-Pierre S (1996) The hyperphagic effect of a high-fat diet and alcohol intake persists after control for energy density. Am J Clin Nutr 63, 479-482.

31. Cummings JR, Ray LA \& Tomiyama AJ (2017) Food-alcohol competition: as young females eat more food, do they drink less alcohol? J Health Psychol 22, 674-683.

32. Eiler W, Dzemidzic M, Case K, et al. (2014) The aperitif effect: alcohol's effects on the brain's response to food aromas. Alcohol Clin Exp Res 38, 236A.

33. Eiler WJ, Džemidžić M, Case KR, et al. (2015) The aperitif effect: alcohol's effects on the brain's response to food aromas in women. Obesity 23, 1386-1393.

34. Kido M, Asakawa A, Koyama KK, et al. (2016) Acute effects of traditional Japanese alcohol beverages on blood glucose and polysomnography levels in healthy subjects. Peerf $\mathbf{4}$, e1853.

35. Kirk JM (1996) Possible factors affecting humans' self-control for food: deprivation level, mood, and alcohol consumption. PhD Thesis, State University of New York at Stony Brook.

36. Kokavec A \& Halloran MA (2011) Red wine alters the glucoseinsulin relationship when consumed alone after a meal. Int J Diab Metab 19, 1-9.
37. Morimoto-Kobayashi Y, Ohara K, Ashigai H, et al. (2015) Matured hop extract reduces body fat in healthy overweight humans: a randomized, double-blind, placebo-controlled parallel group study. Nutr J 15, 25.

38. Polivy J \& Herman CP (1976) Effects of alcohol on eating behavior: influence of mood and perceived intoxication. J Abnorm Psychol 85, 601-606.

39. Caton SJ, Ball M, Ahern A, et al. (2004) Dose-dependent effects of alcohol on appetite and food intake. Physiol Behav 81, 51-58.

40. Caton S, Marks J \& Hetherington M (2005) Pleasure and alcohol: manipulating pleasantness and the acute effects of alcohol on food intake. Physiol Behav 84, 371-377.

41. Caton S, Bate L \& Hetherington M (2007) Acute effects of an alcoholic drink on food intake: aperitif versus co-ingestion. Physiol Behav 90, 368-375.

42. Christiansen P, Abigail A, Randall-Smith L, et al. (2016) Alcohol's acute effect on food intake is mediated by inhibitory control impairments. Health Psychol 35, 518-522.

43. Cordain L, Bryan ED, Melby CL, et al. (1997) Influence of moderate daily wine consumption on body weight regulation and metabolism in healthy free-living males. J Am Coll Nutr 16, 134-139.

44. Cordain L, Melby CL, Hamamoto AE, et al. (2000) Influence of moderate chronic wine consumption on insulin sensitivity and other correlates of syndrome $\mathrm{X}$ in moderately obese women. Metabolism 49, 1473-1478.

45. Hetherington MM, Cameron F, Wallis DJ, et al. (2001) Stimulation of appetite by alcohol. Physiol Behav 74, 283-289.

46. Hofmann W \& Friese M (2008) Impulses got the better of me: alcohol moderates the influence of implicit attitudes toward food cues on eating behavior. J Abnorm Psychol 117, 420-427.

47. Mattes RD (1996) Dietary compensation by humans for supplemental energy provided as ethanol or carbohydrate in fluids. Physiol Behav 59, 179-187.

48. Ouwens MA, van Strien T \& van der Staak CP (2003) Absence of a disinhibition effect of alcohol on food consumption. Eat Behav 4, 323-332.

49. Schrieks IC, Stafleu A, Griffioen-Roose S, et al. (2015) Moderate alcohol consumption stimulates food intake and food reward of savoury foods. Appetite 89, 77-83.

50. Tremblay A, Wouters E, Wenker M, et al. (1995) Alcohol and a high-fat diet: a combination favoring overfeeding. Am J Clin Nutr 62, 639-644.

51. Westerterp-Plantenga MS \& Verwegen CR (1999) The appetizing effect of an aperitif in overweight and normalweight humans. Am J Clin Nutr 69, 205-212.

52. Yeomans MR, Hails N \& Nesic J (1999) Alcohol and the appetizer effect. Behav Pharmacol 10, 151-161.

53. Yeomans MR \& Phillips MF (2002) Failure to reduce short-term appetite following alcohol is independent of beliefs about the presence of alcohol. Nutr Neurosci 5 , 131-139.

54. Yeomans MR (2010) Short term effects of alcohol on appetite in humans. Effects of context and restrained eating. Appetite 55, 565-573.

55. Caton SJ, Nolan LJ \& Hetherington MM (2015) Alcohol, appetite and loss of restraint. Curr Obes Rep 4, 99-105.

56. Lobo IA \& Harris RA (2008) GABA(A) receptors and alcohol. Pharmacol Biochem Behav 90, 90-94.

57. Kumar S, Porcu P, Werner DF, et al. (2009) The role of GABA (A) receptors in the acute and chronic effects of ethanol: a decade of progress. Psychopharmacology (Berl) 205, 529-564. 
58. Widdowson PS \& Holman RB (1992) Ethanol-induced increase in endogenous dopamine release may involve endogenous opiates. J Neurochem 59, 157-163.

59. Yeomans MR \& Gray RW (2002) Opioid peptides and the control of human ingestive behaviour. Neurosci Biobehav Rev 26, $713-728$

60. Office for National Statistics (2017) Adult Drinking Habits in Great Britain: 2005 to 2016. South Wales: Office of National Statistics.

61. Simone P \& Steve C (2006) Consumers' expectations of food and alcohol pairing. Br Food J 108, 169-180.

62. Brown L (2016) Health Survey for England 2015: Adult Alcohol Consumption. Leeds: Health and Social Care Information Centre.

63. Australian Institute of Health and Welfare (2017) National Drug Strategy Household Survey (NDSHS) 2016 - key findings. https://www.aihw.gov.au/reports/illicit-use-ofdrugs/ndshs-2016-key-findings/contents/summary (accessed October 2017).

64. Mitchell Jr MC, Teigen EL \& Ramchandani VA (2014) Absorption and peak blood alcohol concentration after drinking beer, wine, or spirits. Alcohol Clin Exp Res 38, 1200-1204.

65. Finlayson G \& Dalton M (2012) Hedonics of food consumption: are food 'liking' and 'wanting' viable targets for appetite control in the obese? Curr Obes Rep 1, 42-49.

66. Finlayson G, King N \& Blundell JE (2007) Liking vs. wanting food: importance for human appetite control and weight regulation. Neurosci Biobehav Rev 31, 987-1002.

67. Rucker G \& Schumacher M (2008) Simpson's paradox visualized: the example of the rosiglitazone meta-analysis. $B M C$ Med Res Methodol 8, 34 